PAPers in Physics, VOL. 12, ART. 120004 (2020)

Received: 13 August 2019, Accepted: 20 March 2020

Edited by: M. C. Barbosa

Licence: Creative Commons Attribution 4.0

DOI: https://doi.org/10.4279/PIP.120004

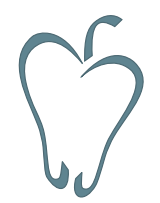

ISSN 1852-4249

\title{
Flavodoxin in a binary surfactant system consisting of the nonionic 1-decanoyl-rac-glycerol and the zwitterionic lauryldimethylamine-N-oxide: Molecular dynamics simulation approach
}

\author{
Behnaz Bazaziyan ${ }^{1}$, Mohammad Reza Bozorgmehr $^{1 *}$, Mohammad Momen-Heravi ${ }^{1}$, \\ S. Ali Beyramabadi ${ }^{1}$
}

\begin{abstract}
Due to the short time constant of the spin-spin relaxation process, there is a limitation in the preparation of NMR sample solution for large proteins. To overcome this problem, reverse micelle systems are used. Here, molecular dynamics simulation was used to study the structure of flavodoxin in a quaternary mixture of 1-decanoyl-rac-glycerol, lauryldimethylamine-N-oxide, pentane and hexanol. Hexanol was used as co-solvent. Simulations were performed at three different co-solvent concentrations. The proportion of components in the mixture was selected according to experimental conditions. For comparison, simulation of flavodoxin in water was also performed. The simulation results show that the $\mathrm{C} \alpha$-RMSD for the protein in water is less than for the surfactant mixture. Also, the radius of gyration of flavodoxin increased in the presence of surfactants. The distance between the two residues trp-57 and phe-94, as a measure of protein activity, was obtained from the simulations. The results showed that in the surfactant mixtures this distance increases. Analysis of the secondary structure of the protein shows that the N-terminal part of the flavodoxin is more affected by surfactants. The flavodoxin diffusion coefficient in the surfactant mixture decreased in relation to its diffusion coefficient in water.
\end{abstract}

\section{Introduction}

Nuclear magnetic resonance (NMR) spectroscopy is a powerful technique for studying the structure of proteins under different conditions [1]. This method is used for high-purity protein samples in an aqueous phase. In the case of spin-spin relaxation, the transverse component of the magnetization vector decays exponentially towards its equilibrium value in NMR. The time constant describing this process is known as $T_{2}$. This name is based on $T_{1}$, which is the time constant of the spin-lattice

* bozorgmehr@mshdiau.ac.ir

1 Department of Chemistry, Mashhad Branch, Islamic Azad University, Mashhad, Iran. relaxation process. The short $T_{2}$ time causes pulse loss during pulse sequences. On the other hand, with increasing protein size, this time is reduced. It is therefore difficult to prepare a solution for large proteins, so using this method for them is limited. By encapsulating the protein in the aqueous solution formed in the cavity of the reverse micelles, and then placing the complex in a non-polar solvent, this problem is largely resolved [2]. In this case, the viscosity of the non-polar solvent must be much less than water [3]. Short chain alkanes are a good candidate for this purpose [4]. The nature of surfactants, the ability to dissolve surfactant in a non-polar solvent, and the ratio of water to surfactant are among the important parameters for the success of this method [5]. 
Papers in Physics, vol. 12, ART. 120004 (2020) / B. Bazaziyan et al.

Flavodoxin is an electron transfer protein that plays a role in photosynthetic reactions [6]. In flavodoxins, a Flavin mono nucleotide molecule is attached to the protein in a non-covalent manner, which causes the redox property of proteins [7]. From a structural standpoint, flavdoxin is an $\alpha / \beta$ protein in which the $\beta$ sheets are surrounded by $\alpha$ helices [8]. Since flavodoxin is a simple onedomain protein, it is a suitable model for protein studies such as stability and folding. Cheung et al. have investigated the dynamics of flavodoxin protein in crowded environments by molecular dynamics simulation [9]. They showed that protein stability increases in in silico crowding conditions, and folding routes experiencing topological frustrations can be either relieved or enhanced upon manipulation of the crowding conditions. Studies have also shown that by removing the Flavin mononucleotide molecule from flavodoxin and creating the Apoprotein form, the binding position does not change greatly; only the aromatic rings of tyrosine-94 and tryptophan-57 are closer to each other [6]. Genzor et al. studied the stability of apo-flavodoxin conformation in urea solution in different acidic and ionic strengths. They found that at a $\mathrm{pH}$ of between 6 and 11 apo-flavodoxin conformation is stable, whereas at a lower $\mathrm{pH}$, between 2 and 3.5, proteins lose their stability [10]. The structure of flavodoxin in the presence of a cross-linked, spherical, sucrose-based polymer called Ficoll 70 has been studied experimentally and theoretically $[11,12]$. This circular dichroism and in silico study showed that the helical content of folded apoflavodoxin was increased by additions of Ficoll 70. Other experimental evidence indicated that correct contact formation around the third $\beta$-strand of flavodoxin in the central sheet is crucial to continued folding to the native state in crowded media. Research has also shown that flavodoxin in the presence of Dextran retains about $70 \%$ of its secondary structure [13].

In this manuscript, a simulation of molecular dynamics was used to study the flavdoxin protein in a mixture of the surfactants 1-decanoyl-rac-glycerol and lauryldimethylamine-N-oxide. Pentane solvent was used for solute solvation. For comparison, molecular dynamics simulation of protein in water was also performed.

\section{Computational details}

For the molecular dynamics simulation, four simulation boxes were designed. In the first box, the box dimensions were considered according to the size of the flavodoxin, $5.95 \times 5.95 \times 5.95 \mathrm{~nm}^{3}$, and the protein was placed in the center of the box. Then the box was filled with water solvent (named as System A). In the second box, the protein was placed in the center of the box, with dimensions $6.75 \times 6.75 \times 6.75 \mathrm{~nm}^{3}$, then 1301 decanoyl-rac-glycerol molecules and 70 molecules of lauryldimethylamine-N-oxide were randomly added to the box. Also, 50 molecules of water were added to this box, and finally, the box was filled with 1000 pentane molecules (named as System B). The specification of the third box was like the second box, only differing in that 5 hexanol molecules were also randomly placed in the box (named as System $\mathbf{C}$ ). The fourth box was similar to the second box, but in this box, 10 hexanol molecules were randomly placed in it (named as System $\mathbf{D}$ ). The components used in the various systems are summarized in Table 1.

Since 1-decanoyl-rac-glycerol is non-ionic, hexanol solvent is used as auxiliary solvent. These molecular ratios are selected based on the reported optimal ratios in Dodevski et al.'s experimental work [14]. The structure of flavodoxin was obtained from the protein data bank with code 1obv [15]. To perform molecular dynamics simulation, the Gromacs software version 5.2.1 was used [16]. Charm27 all-atom force field was used for calculations, since the force field parameters for 1-decanoyl-rac-glycerol, lauryldimethylamine$\mathrm{N}$-oxide, hexanol and pentane are not present in the default version of the Gromacs software. The structure of these compounds was optimized using the B3LYP density functional method with the basis function of $6-31 \mathrm{G}^{*}$. To control the optimization, frequency calculations were performed and virtual frequencies were not observed. The charge of each atom was calculated by the CHELPG method. All ab initio calculations were done by GAMESS software package [17]. The optimized structure of the compounds is shown in Fig. 1.

The SwissParam web server [18] was used to determine the force field parameters of the studied compounds. Water model TIP3P [19] was used for water in simulation boxes. In order to neutralize 
Papers in Physics, vol. 12, ART. 120004 (2020) / B. Bazaziyan et al.

Table 1: Outline of designed systems.

\begin{tabular}{cccccc}
\hline \hline System & box volume $\left(\mathrm{nm}^{3}\right)$ & 1-decanoyl-rac-glycerol & lauryldimethylamine-N-oxide & pentane & hexanol \\
\hline $\mathbf{A}$ & $5.95 \times 5.95 \times 5.95$ & - & - & - & - \\
$\mathbf{B}$ & $6.75 \times 6.75 \times 6.75$ & 130 & 70 & 1000 & - \\
$\mathbf{C}$ & $6.75 \times 6.75 \times 6.75$ & 130 & 70 & 1000 & 5 \\
$\mathbf{D}$ & $6.75 \times 6.75 \times 6.75$ & 130 & 70 & 1000 & 10 \\
\hline \hline
\end{tabular}

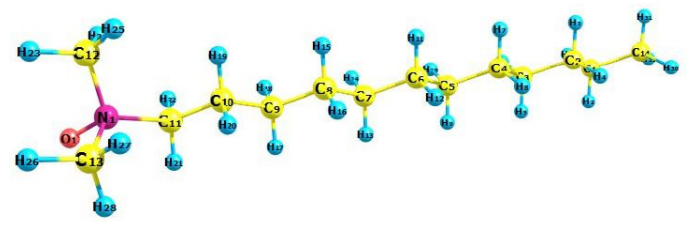

1-decanoyl-rac-glycerol

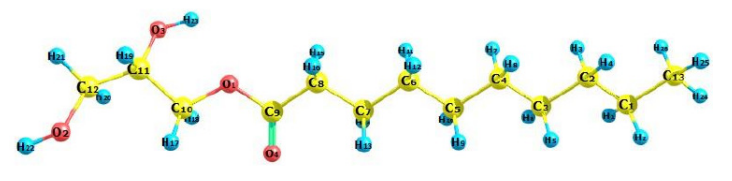

lauryldimethylamine-N-oxide
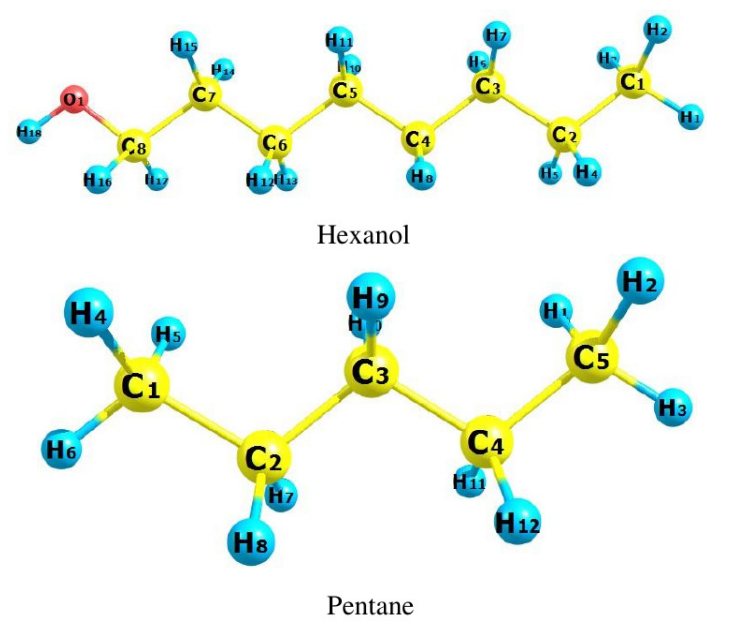

Figure 1: Optimized geometries of studied molecules, with labeling.

the systems, an appropriate number of $\mathrm{Na}^{+}$ions were added to each box. To fix a constant temperature and pressure during the simulations, system components were coupled with $\mathrm{V}$-rescale and Nose-Hoover thermostat [20] respectively, in each of the equilibration steps and molecular dynamics simulations. The temperature and pressure used in the calculations are 298 Kelvin and 1 bar, re- spectively. LINCS algorithm [21] was employed to fix the chemical bonds between the atoms of the non-water molecules and SETTLE algorithm [22] was used in the case of water molecules. The PME algorithm was used to calculate electrostatic interactions [23]. Energy minimization was done using the steepest descent method [24] to eliminate the primary kinetic energy and to eliminate inappropriate contacts between the atoms. Each simulation box achieved a two-stage equilibrium in NVT and NPT ensemble. At this stage, the time of equilibration was considered 10 ns with a time step of 2 fs. Finally, molecular dynamics was performed by solving the second Newton equation for 140 ns with the 2 fs time step. Each simulation was repeated four times under the different initial conditions, to avoid any dependency on the initial conditions and to increase the accuracy of the simulations.

\section{Results and discussion}

An important point when using surfactants in encapsulating proteins in NMR spectroscopy is to maintain the native protein structure and reduce its reorientation time. Therefore, the structural and dynamic parameters of flavodoxin in the designed systems were calculated for analysis. One common method of displaying the stability of a protein's structure is to calculate the RMSD values during the simulation. The root mean square deviation (RMSD) is defined as:

$$
\operatorname{RMSD}\left(t_{1}, t_{2}\right)=\left[\frac{1}{M} \sum_{i=1}^{N} m_{i}\left|\mathbf{r}_{i}\left(t_{1}\right)-\mathbf{r}_{i}\left(t_{2}\right)\right|\right]^{\frac{1}{2}}
$$

Where $\mathbf{r}_{i}$ is the atomic position at time $t$ and $M=$ $\sum_{i=1}^{N} m_{i}$. C $\alpha$-RMSD variations of flavodoxin for the studied systems are shown in Fig. 2. 
PApers In Physics, vol. 12, ART. 120004 (2020) / B. Bazaziyan et al.

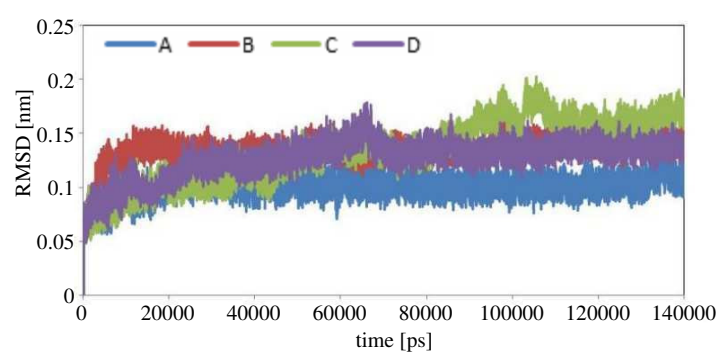

Figure 2: $\mathrm{C} \alpha$-RMSD values (nm) versus time (ps) for simulated systems defined as $\mathbf{A}, \mathbf{B}, \mathbf{C}$ and $\mathbf{D}$.

According to the figure, protein stability in surfactant systems is observed to be somewhat reduced compared to system A. This decrease in stability for the $\mathbf{C}$ system is greater than for $\mathbf{B}$ and $\mathbf{D}$. However, the difference in $\mathrm{C} \alpha$-RMSD values in different systems is not high. On the other hand, given the fact that flavodoxin has 169 residues, this difference in stability in various systems is not significant. Another factor that can determine the activity and stability of the protein is compression of the conformation [25], in which the radius of gyration $\left(R_{g}\right)$ indicates the compression of the protein structure. The $R_{g}$ of a protein is calculated using the following equation:

$$
R_{g}=\left(\frac{\sum_{i}\left|\mathbf{r}_{i}\right|^{2} m_{i}}{\sum_{i} m_{i}}\right)^{\frac{1}{2}}
$$

Where $m_{i}$ is the atomic mass of $i$ and $\mathbf{r}_{i}$ the atomic position of $i$ relative to the center of mass of the molecule [26]. The $R_{g}$ of the studied systems was calculated and the results are shown in Fig. 3.

The $R_{g}$ values of the proteins vary from $1.48 \mathrm{~nm}$ to $1.63 \mathrm{~nm}$ in different systems. Flavodoxin in system $\mathbf{A}$ has the smallest $R_{g}$ and in system $\mathbf{C}$ the highest $R_{g}$. Since the compactness of the structure of the proteins is attributed to their activity [27], according to 3 the presence of flavodoxin in the surfactant solution decreases its activity. The root mean square fluctuation (RMSF) values of flavodoxin sequences are calculated in different systems and shown in Fig. 4.

Except for the residues valine-18, isoleucine-21,

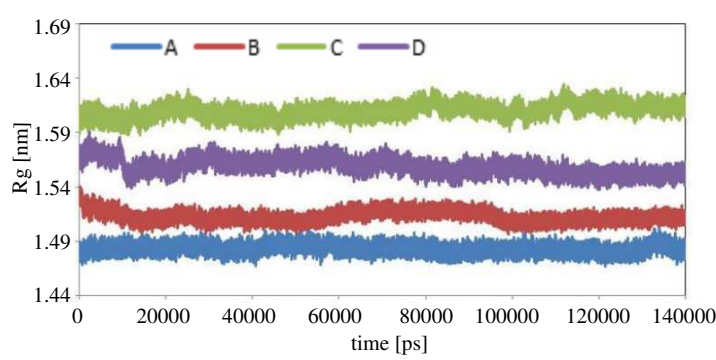

Figure 3: $R_{g}$ values (nm) versus time (ps) for simulated systems defined as $\mathbf{A}, \mathbf{B}, \mathbf{C}$ and $\mathbf{D}$.

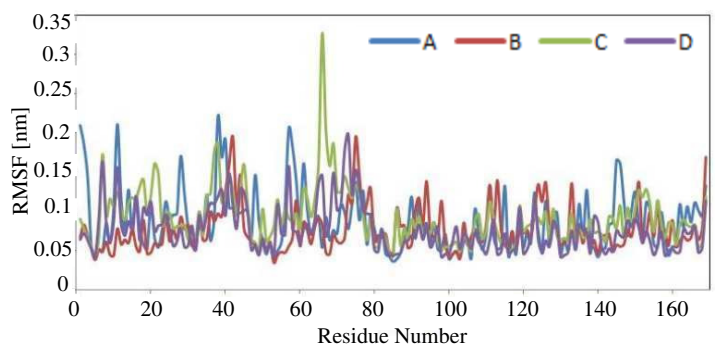

Figure 4: Average RMSF values for each residue of Flavodoxin during simulation in $\mathbf{A}, \mathbf{B}, \mathbf{C}$ and $\mathbf{D}$ systems.

tryptophan-66 and glycine- 68 in the flavodoxin of system $\mathbf{C}$, the flexibility of other residues in system A is more than in the other systems. Another noteworthy point is that the $\mathrm{C}$-terminal of the protein is less affected by the non-aqueous environment. In fact, the greatest difference in the RMSF values in the various systems related to amino acids is 1 to 79 . In this region, the helices and sheets in the secondary structure of flavodoxin have smaller lengths than the helices and sheets in the c-terminal of the protein. If two faces are considered for proteins, the face of the N-terminal is more exposed to the environment. In flavodoxins, the distance between two aromatic amino acids in the ligand binding site is considered as a measure of protein activity [8]. The distance between the tryptophan57 and phenylalanine-94 residues of flavodoxin in 


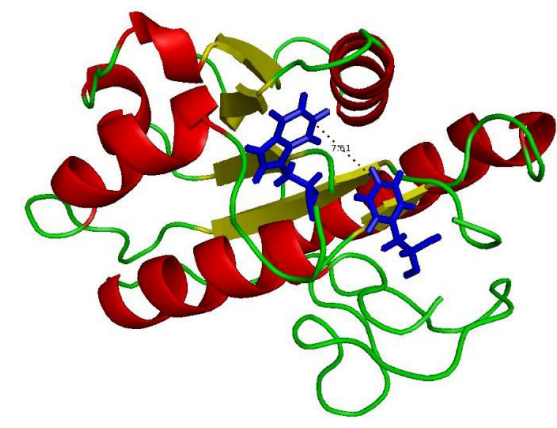

Figure 5: The distance between Trp-57 and Phe-94 (system A).

system $\mathbf{A}$ is shown in Fig. 5 .

According to the figure, this distance is 7.61 angstroms. The distance between these two amino acids in systems $\mathbf{B}, \mathbf{C}$ and $\mathbf{D}$ was $9.48,10.05$ and 10.42 angstroms, respectively. According to the values obtained, flavdoxin activity seems to decrease in surfactant solutions. Sampling of the suitable structure for analysis in molecular dynamics trajectories has always been one of the interesting issues. Averaging the coordinates of the molecular coordinates in the last few nanoseconds of the simulation is one of the methods used in this regard [28]. The sampling of coordinates at a time when the system is in equilibrium is another method used for this [29]. In these methods, variations of a quantity such as $\mathrm{C} \alpha$-RMSD in the area are used. However, there is no physical concept in the average of atomic coordinates [30], so for sampling of the simulationsthe free energy landscape (FEL) analysis method is used [31]. There are three main stages of FEL analysis: calculation of the $\mathrm{C} \alpha$-RMSD and the flavodoxin radius of gyration $\left(R_{g}\right)$, obtaining the possibility of the presence of flavodoxin protein conformation in each corresponding value of $\mathrm{C} \alpha$-RMSD and $R_{g}$, and calculation of the free energy of configurations based on the probability values of presence. The results of the FEL analysis are shown in 3D diagrams in Fig. 6 for $\mathbf{A}, \mathbf{B}, \mathbf{C}$ and $\mathbf{D}$ systems, respectively.

In these figures, the minimum regions of free energy are shown in blue. According to these figures, it can be seen that all systems have a local minimum with the least amount of free energy. Regarding the values of $\mathrm{C} \alpha$-RMSD and $R_{g}$ corresponding

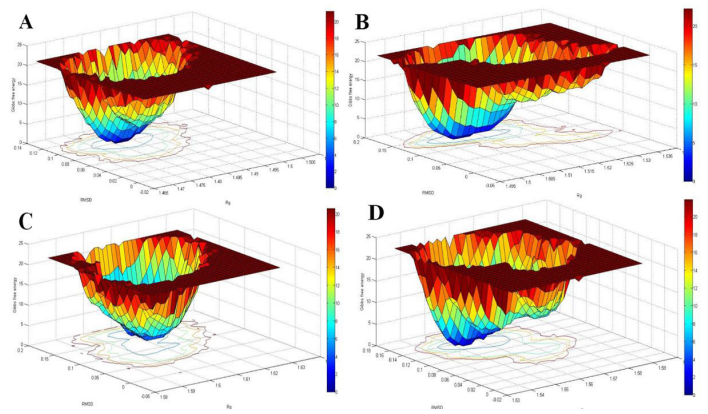

Figure 6: Free Energy Landscape of the A, B, C and D simulated systems.

to the lowest free energy, the structure of flavodoxin was sampled from the molecular trajectories in the simulations. Then, using the software polyview 2D [32], the secondary structure of the flavodoxin obtained in the previous step was analyzed. The result is shown in Fig. 7 for different systems.

As the color darkens, it indicates that the level of solvent-accessible surface area for the residue decreases. With respect to the figures, it is observed that in the $\mathbf{B}$ system, the helices between aspartic-35 and aspartic-46 are eliminated. The beta sheet between the Valin-31 and Aspartic-35 sequences has also been shortened. The downsizing of this beta sheet in $\mathbf{C}$ and $\mathbf{D}$ systems is less than in the $\mathbf{B}$ system. In these areas, by changing the structure of the helices and sheets, the level of solvent-accessible surface area of residues has increased somewhat. The region of change for the $\mathbf{C}$ system is related to the helices between the tryptophan- 57 and the aspartic- 75 residues. In general, the secondary structure of the flavodoxin in the c-terminal region has changed little. These results are consistent with the results of the RMSF values.

A protein contact map is a convenient way to determine changes in the tertiary structure of proteins in different conditions. The flavodoxin contact map was obtained in various simulations and the results are shown in Fig. 8. In these figures, the flavodoxin structure of system $\mathbf{A}$ was considered as a wild structure, and the remaining structures were compared to system $\mathbf{A}$. In these figures, the lower half is the contact map of flavodoxin, while the upper half is the protein difference contact map; they 
PAPers In Physics, vol. 12, ART. 120004 (2020) / B. Bazaziyan et al.
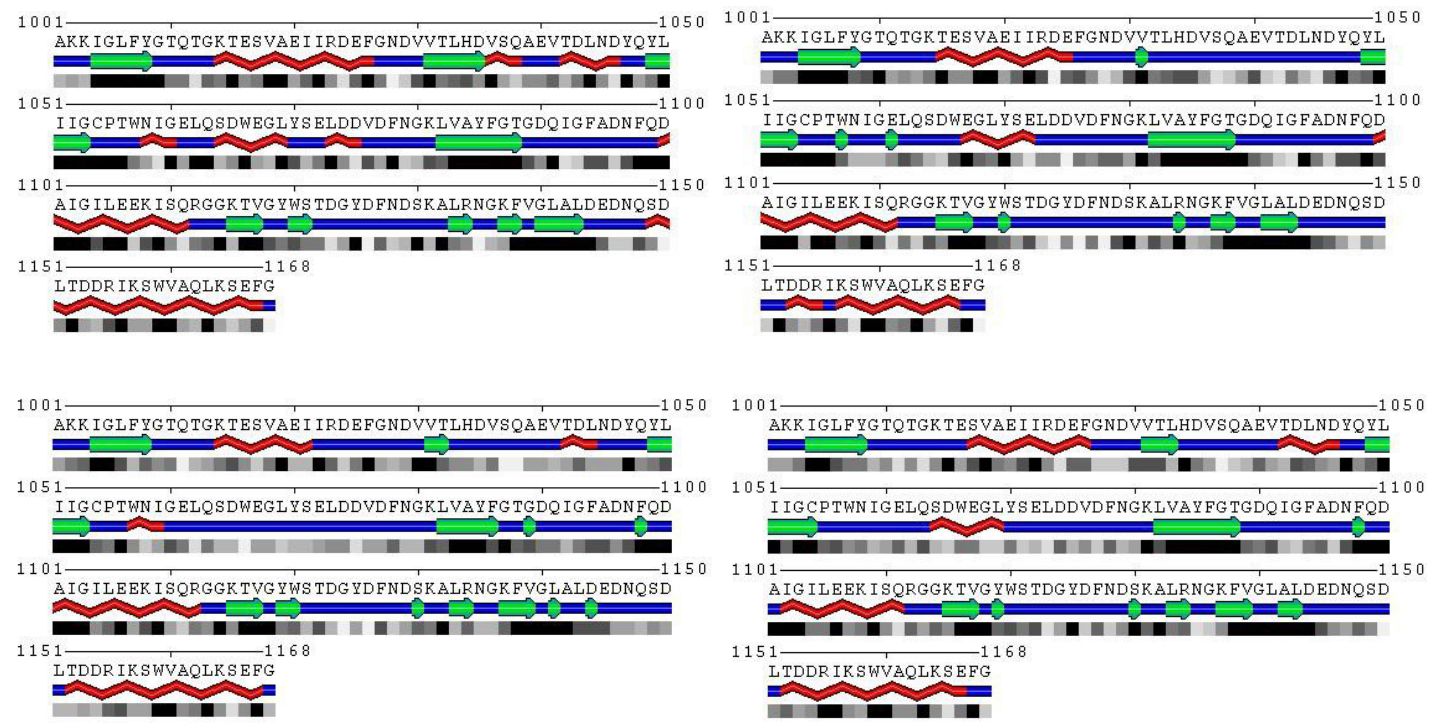

$\approx$ Helix, $\Longrightarrow \beta$ strand, $\rightleftharpoons$ coil.

Dark: completely buried, bright: completely exposed

Figure 7: The secondary structure of the Flavodoxin, along with the solvent-accessible surface area for its residues in systems $\mathbf{A}$ (top-left), $\mathbf{B}$ (top-right), $\mathbf{C}$ (bottom-left) and $\mathbf{D}$ (bottom-right).
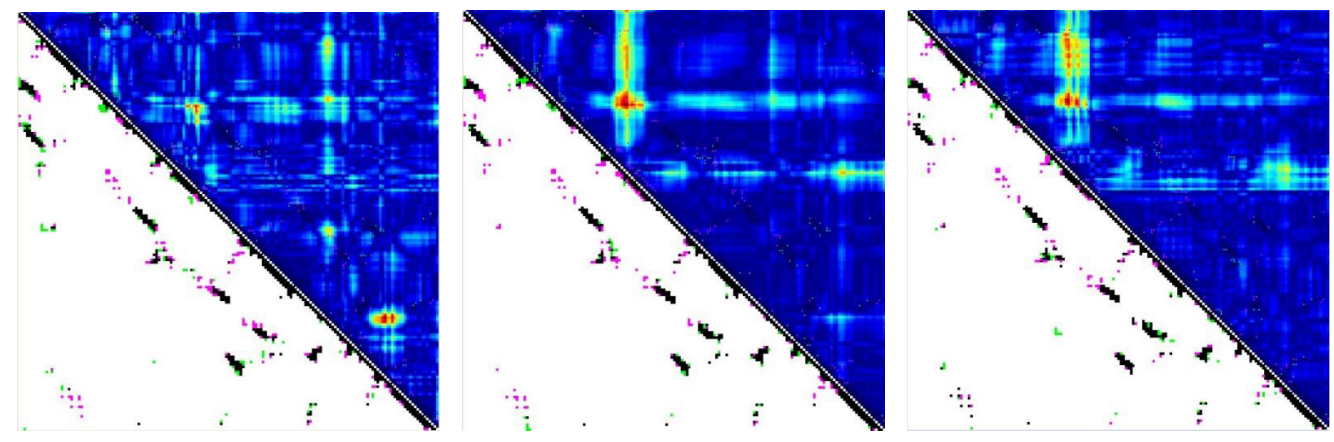

Figure 8: (Left) The contact map of Flavodoxin in B (left-lower triangle) and different contact map of Flavodoxin in $\mathbf{B}$ and Flavodoxin in $\mathbf{A}$ (right-upper triangle). (Center) The contact map of Flavodoxin in $\mathbf{C}$ (left-lower triangle) and different contact map of Flavodoxin in $\mathbf{C}$ and Flavodoxin in A (right-upper triangle). (Right) The contact map of Flavodoxin in $\mathbf{D}$ (left-lower triangle) and different contact map of Flavodoxin in $\mathbf{D}$ and Flavodoxin in A (right-upper triangle). The colors used in the figure are described in the main text.

are shown in different systems relative to system $\mathbf{A}$. In the lower part of the figure, the black color represents the common contacts and the green color indicates the contacts that are in the wild structure of system A, but not in the structure of the secondary system; the red color represents the contacts that are in the secondary structure but not in the structure of system A. In the upper part, the blue color indicates the regions with the smallest differences and the red color indicates the areas with the largest differences. The greater the difference in red color between Figs. 8 (center) and 8 (right) in comparison with Fig. 8 (left), the more the tertiary structure of flavodoxin in systems $\mathbf{C}$ and $\mathbf{D}$ has been affected. Also, areas of tertiary structure change in these systems are more focused 
PApers in Physics, vol. 12, ART. 120004 (2020) / B. Bazaziyan et al.

than in system B. Finally, to determine the dynamics of flavodoxin, its diffusion coefficient was calculated. Prediction of the diffusion coefficients of molecules in solvent is of theoretical importance. As the diffusion coefficient value increases, the mobility of the molecule increases [33]. The Einstein relation was used to calculate the diffusion coefficient of flavodoxin in all simulated systems:

$$
D=\frac{1}{6} \lim _{t \rightarrow \infty} \frac{d}{d t}\left\langle\left|\mathbf{r}_{i}(t)-\mathbf{r}_{i}(0)\right|^{2}\right\rangle .
$$

Where $\mathbf{r}_{i}$ is the atom coordinate vector and the term inside the angled brackets is the mean square displacement (MSD). In this approach, the selfdiffusion coefficient is proportional to the slope of the MSD as a function of time in the diffusional regime [34]. The diffusion coefficient values of flavodoxin are listed in Table 2.

Table 2: Diffusion coefficient values of Flavodoxin

\begin{tabular}{cc}
\hline \hline System & $D \times 10^{-5} \mathrm{~cm}^{2} / \mathrm{s}$ \\
\hline A & 0.086 \\
B & 0.004 \\
C & 0.004 \\
D & 0.016 \\
\hline \hline
\end{tabular}

With regard to the diffusion coefficient, the flavodoxin dynamics are slower in systems $\mathbf{B}, \mathbf{C}$ and $\mathbf{D}$ than in system $\mathbf{A}$. Thus, the time of reorientation of protein conformation in systems $\mathbf{B}, \mathbf{C}$ and $\mathbf{D}$ is less than that of system $\mathbf{A}$. In other words, using this surfactant mixture can lead to a better NMR spectrum. This result is consistent with the results in the experimental work of Dodevski et al [14].

\section{Conclusions}

By encapsulating the protein in the aqueous solution formed in the cavity of the reverse micelles, and then placing the complex in a non-polar solvent, the problem of slow tumbling will be greatly resolved in NMR spectroscopy. In this work, a mixture of the surfactants 1-decanoyl-rac-glycerol and lauryldimethylamine-N-oxide were used, together with the auxiliary solvent hexanol. Changes in the structure and dynamics of flavodoxin protein were studied in this mixture. Pentane solvent was used as a low viscosity solvent. Compared with the structure of flavodoxin in water, the simulation results indicate that the protein's secondary and tertiary structures in the mixture of surfactants are altered. However, its dynamics decrease. It was observed that in the absence of hexanol the helical and sheet content of the protein decreased in different regions. Also, in the presence of hexanol the ordered secondary structure of the protein decreased relative to two component systems consisting of protein and water. However, the level of reduction of the regular secondary structure was lower than in the absence of hexanol. Decreasing the areas of the regular secondary structure made the sequences more accessible to solvent. In general, the secondary structure of the flavodoxin in the c-terminal region changed little. The different data obtained from the simulation are consistent with each other. The results are also in agreement with previous work in this area [35].

[1] A D Bax, S Grzesiek, Methodological advances in protein NMR, Acc. Chem. Res. 26, 131 (1993).

[2] A J Wand, M R Ehrhardt, P F Flynn, Highresolution NMR of encapsulated proteins dissolved in low-viscosity fluids, Proc. Natl. Acad. Sci. U.S.A. 95, 15299 (1998).

[3] M R Ehrhardt, P F Flynn, A J Wand, Preparation of encapsulated proteins dissolved in low viscosity fluids, J. Biomol. NMR 14, 75 (1999).

[4] C R Babu, P F Flynn, A J Wand, Validation of protein structure from preparations of encapsulated proteins dissolved in low viscosity fluids, J. Am. Chem. Soc. 123, 2691 (2001).

[5] W D Van Horn, M E Ogilvie, P F Flynn, Use of reverse micelles in membrane protein structural biology, J. Biomol. NMR 40, 203 (2008).

[6] J Sancho, Flavodoxins: Sequence, folding, binding, function and beyond, Cell. Mol. Life Sci. 63, 855 (2006).

[7] K Fukuyama, H Matsubara, L J Rogers, Crystal structure of oxidized flavodoxin from a red alga Chondrus crispus refined at $1.8 \AA$ resolution: Description of the flavin mononucleotide binding site, J. Mol. Biol. 225, 775 (1992). 
Papers in Physics, vol. 12, ART. 120004 (2020) / B. Bazaziyan et al.

[8] S Maldonado, A Lostao, M P Irún, J Férnandez-Recio, C G Genzor, E B González, J A Rubio, A Luquita, F Daoudi, J Sancho, Apoflavodoxin: Structure, stability, and FMN binding, Biochimie 80, 813 (1998).

[9] D Homouz, L Stagg, P Wittung-Stafshede, M S Cheung, Macromolecular crowding modulates folding mechanism of $\alpha / \beta$ protein apoflavodoxin, Biophys. J. 96, 671 (2009).

[10] C G Genzor, C Gómez-Moreno, J Sancho, A Beldarraín, J L López-Lacomba, M Cortijo, Conformational stability of apoflavodoxin, Protein Sci. 5, 1376 (1996).

[11] L Stagg et al, Molecular crowding enhances native structure and stability of $\alpha / \beta$ protein flavodoxin, Proc. Natl. Acad. Sci. U.S.A. 104, 18976 (2007).

[12] D Venturoli, B Rippe, Ficoll and dextran vs. globular proteins as probes for testing glomerular permselectivity: Effects of molecular size, shape, charge, and deformability, Am. J. Physiol. Renal Physiol. 288, F605 (2005).

[13] E Steensma, C P van Mierlo, Structural characterisation of apoflavodoxin shows that the location of the stable nucleus differs among proteins with a flavodoxin-like topology, J. Mol. Biol. 282, 653 (1998).

[14] I Dodevski, N V Nucci, K G Valentine, G K Sidhu, E S O'Brien, A Pardi, A J Wand, Optimized reverse micelle surfactant system for high-resolution NMR spectroscopy of encapsulated proteins and nucleic acids dissolved in low viscosity fluids, J. Am. Chem. Soc. 136 , 3465 (2014).

[15] A Lostao, F Daoudi, M P Irún, Á Ramón, C Fernández-Cabrera, A Romero, J Sancho, How FMN Binds to Anabaena Apoflavodoxin: $A$ hydrophobic encounter at an open binding site, J. Biol. Chem. 278, 24053 (2003).

[16] D Van Der Spoel, E Lindahl, B Hess, G Groenhof, A E Mark, H J Berendsen, GROMACS: Fast, flexible, and free, J. Comput. Chem. 26, 1701 (2005).
[17] M W Schmidt, K K Baldridge, J A Boatz, S T Elbert, M S Gordon, J H Jensen, S Koseki, N Matsunaga, K A Nguyen, S Su, General atomic and molecular electronic structure system, J. Comput. Chem. 14, 1347 (1993).

[18] V Zoete, M A Cuendet, A Grosdidier, O Michielin, SwissParam: A fast force field generation tool for small organic molecules, J. Comput. Chem. 32, 2359 (2011).

[19] D J Price, C L Brooks III, A modified TIP3P water potential for simulation with Ewald summation, J. Chem. Phys. 121, 10096 (2004).

[20] G Bussi, D Donadio, M Parrinello, Canonical sampling through velocity rescaling, J. Chem. Phys. 126, 014101 (2007).

[21] B Hess, H Bekker, H J Berendsen, J G Fraaije, LINCS: A linear constraint solver for molecular simulations, J. Comput. Chem. 18, 1463 (1997).

[22] S Miyamoto, P A Kollman, SETTLE: An analytical version of the SHAKE and RATTLE algorithm for rigid water models, J. Comput. Chem. 13, 952 (1992).

[23] U Essmann, L Perera, M L Berkowitz, T Darden, H Lee, L G Pedersen, A smooth particle mesh Ewald method, J. Chem. Phys. 103, 8577 (1995).

[24] D G Luenberger, Y Ye, Linear and nonlinear programming, Springer International Publishing, Switzerland (1984).

[25] S Ghaderi, M R Bozorgmehr, A Morsali, Structure study and predict the function of the diphtheria toxin in different $p H$ levels (AcidicBasic-Natural) using molecular dynamics simulations, Entomol. Appl. Sci. Lett. 3, 49 (2016).

[26] B Honarparvar, A A Skelton, Molecular dynamics simulation and conformational analysis of some catalytically active peptides, J. Mol. Model. 21, 100 (2015).

[27] H Monhemi, M R Housaindokht, M R Bozorgmehr, M S S Googheri, Enzyme is stabilized by a protection layer of ionic liquids in 
Papers in Physics, vol. 12, ART. 120004 (2020) / B. Bazaziyan et al.

supercritical CO2: Insights from molecular dynamic simulation, J. Supercrit. Fluids 69, 1 (2012).

[28] M R Housaindokht, M R Bozorgmehr, H E Hosseini, R Jalal, A Asoodeh, M Saberi, Z Haratipour, H Monhemi, Structural properties of the truncated and wild types of Takaamylase: A molecular dynamics simulation and docking study, J. Mol. Catal. B: Enzym. 95, 36 (2013).

[29] N Moghtaderi, M R Bozorgmehr, A Morsali, The study of self-aggregation behavior of the bilirubin molecules in the presence and absence of carbon nanotubes: Molecular dynamics simulation approach, J. Mol. Liq. 208, 342 (2015).

[30] D van der Spoel, E Lindahl, B Hess, A Van Buuren, E Apol, P Meulenhoff, D Tieleman, A Sijbers, K Feenstra, R van Drunen, GROMACS user manual version 3.3, (2008).
[31] H Lei, C Wu, H Liu, Y Duan, Folding freeenergy landscape of villin headpiece subdomain from molecular dynamics simulations, Proc. Natl. Acad. Sci. U.S.A. 104, 4925 (2007).

[32] A A Porollo, R Adamczak, J Meller, POLYVIEW: A flexible visualization tool for structural and functional annotations of proteins, Bioinformatics 20, 2460 (2004).

[33] D Brune, S Kim, Predicting protein diffusion coefficients, Proc. Natl. Acad. Sci. U.S.A. 90, 3835 (1993).

[34] D Frenkel, B Smit, Understanding molecular simulation: From algorithms to applications, Computacional Sciences Series 1, Academic Press (2002).

[35] B Bazaziyan et al, Reverse micelle surfactant system comprising the 1-decanoyl-rac-glycerol and the Lauryldimethylamine-N-oxide: Structure and dynamics of confined water, Russ. J. Phys. Chem. A 93, 1122 (2019). 